\title{
Protumoral roles of melanoma inhibitory activity 2 in oral squamous cell carcinoma
}

\author{
M Kurihara ${ }^{1,2}$, T Kirita ${ }^{2}$, T Sasahira ${ }^{1}$, H Ohmori ${ }^{1}$, S Matsushima ${ }^{1}$, K Yamamoto $^{2}$, A K Bosserhoff ${ }^{3}$ \\ and $\mathrm{H}$ Kuniyasu*,1 \\ ${ }^{1}$ Department of Molecular Pathology, Nara Medical University, 840 Shijo-cho, Kashihara, Nara 634-8521, Japan; ${ }^{2}$ Department of \\ Oral and Maxillofacial Surgery, Nara Medical University, Kashihara, Japan and ${ }^{3}$ Department of Molecular Pathology, Institute of \\ Pathology, University of Regensburg, Regensburg, Germany
}

Background: The role of melanoma inhibitory activity 2 (MIA2) was examined in human oral squamous cell carcinoma (OSCC).

Methods: MIA2 role was examined by immunohistochemistry of human OSCCs and knockdown studies using human 3 OSCC cell lines with MIA2 expression.

Results: MIA2 expression was observed in 62 (66.7\%) of 93 OSCCs and was associated with tumour expansion and nodal metastasis. Melanoma inhibitory activity 2 expression was inversely correlated with intratumoral infiltration of lymphocytes. Invasion and anti-apoptotic survival were reduced by MIA2 knockdown in HSC3 cells. MOLT-3 lymphocytes infiltrating the HSC3 cell layer was enhanced by MIA2 knockdown or MIA2 depletion with the antibody. In HSC3 cells, MIA2 knockdown decreased the expressions of vascular endothelial growth factor (VEGF), VEGF-C, and VEGF-D. The downregulation of VEGF-C and -D was caused by inhibition of p38 and extracellular signal-regulated kinase (ERK)1/2, respectively. Melanoma inhibitory activity 2 was co-precipitated with integrin $\alpha 4$ and $\alpha 5$ in HSC3 cells. Integrin $\alpha 4$ knockdown decreased p38 phosphorylation and increased apoptosis, whereas integrin $\alpha 5$ knockdown decreased c-Jun N-terminal kinase (JNK) phosphorylation and apoptosis. Inhibition of JNK decreased apoptosis in the HSC3 cells.

Conclusion: These findings suggest that the roles of MIA2 might be based on the variety of the integrins and the subtypes of mitogen-activated protein kinase.

Oral squamous cell carcinoma (OSCC) is the most common cancer resulting from the oral habits of smoking, reverse smoking, and betel nut chewing, accounting for at least $40 \%$ of cancer cases in South Asia, including India and Sri Lanka (Ferlay et al, 2010). In Japan, OSCC comprises $\sim 1-2 \%$ of all cancer morbidity, a rate that is gradually increasing (Matsuda et al, 2012). Oral squamous cell carcinoma frequently leads to dysfunction of mastication, speech, and deglutition, which worsen a patient's quality of life. Moreover, the prognosis of OSCC remains poor: the overall 5-year survival rate has remained at $\sim 56 \%$ over the past 2 decades (Nagler, 2002; Kademani et al, 2005). The poor prognosis of OSCC is associated with local invasion and lymph node metastasis (Lopez-Graniel et al, 2001).

Angiogenesis and lymphangiogenesis, pivotal events in tumour progression and metastasis (Adams and Alitalo, 2007; Avraamides et al, 2008), are responsible for local invasion and lymph node metastasis and a subsequently worse prognosis (Miyahara et al, 2007). Tumour cells have been reported to induce angiogenesis and lymphangiogenesis by expressing angiogenesis factor vascular endothelial growth factor (VEGF) and the lymphangiogenic factors VEGF-C and VEGF-D (Beck and D'Amore, 1997; Tsurusaki et al, 1999; Yonemura et al, 1999). In OSCC, like in other tumours, VEGF predominantly induces tumour angiogenesis, whereas VEGF-C and VEGF-D induce lymphangiogenesis (Shintani et al, 2004). A significant association between elevated VEGF circulating levels and clinical stage (Shang et al, 2002), and a possible relationship between the VEGF-C and/or VEGF-D expression levels and the development of lymphatic tumour spread were revealed in patients with OSCC (Shintani et al, 2004). We previously reported that melanoma inhibitory activity (MIA) is

*Correspondence: Dr H Kuniyasu; E-mail: cooninh@zb4.so-net.ne.jp

Received 10 October 2012; revised 2 January 2013; accepted 7 January 2013; published online 19 March 2013

(c) 2013 Cancer Research UK. All rights reserved 0007-0920/13 
closely involved in tumour progression and nodal metastasis by the increments of VEGF-C and VEGF-D in OSCC (Sasahira et al, 2010).

Melanoma inhibitory activity 2 (MIA2) belongs to the MIA gene family, which contains MIA, OTOR, and Tango (Bosserhoff and Buettner, 2002). Melanoma inhibitory activity 2 is mapped to the gene locus of human chromosome 14q13 (Bosserhoff et al, 2003b). Melanoma inhibitory activity and OTOR are exclusively expressed in the cartilage and cochlea, respectively, whereas MIA2 is expressed exclusively in the liver (Bosserhoff et al, 2003b). Melanoma inhibitory activity 2 expression is transcriptionally regulated by the hepatocyte nuclear factor (HNF)-1binding site (Hellerbrand et al, 2005). Melanoma inhibitory activity 2 is expressed in hepatocellular carcinoma (HCC) but not in bladder, breast, or prostate cancer (Hellerbrand et al, 2005). Melanoma inhibitory activity 2 inhibits HCC growth and invasion, and consequently acts as a tumour suppressor (Hellerbrand et al, 2008). Hepatic MIA2 expression is increased in patients with liver fibrosis or cirrhosis and tumour growth factor- $\beta$ (TGF- $\beta$ )-induced MIA2 expression in hepatocytes (Bosserhoff et al, 2003a). It is well known that TGF- $\beta$ signalling is deranged in HCC (Hellerbrand et al, 2008). Therefore, it may be speculated that TGF- $\beta$ signalling contributes to the reduced MIA2 transcriptional activity observed in HCC. Loss of HNF-1 expression in HCC affects tumorigenicity by downregulating MIA2 (Hellerbrand et al, 2008). In OSCC, the expression and role of MIA2 have not been elucidated previously.

In this study, we investigated the relationship between MIA2 expression and clinicopathological characteristics to determine its functional role in OSCC.

\section{MATERIAL AND METHODS}

Patients and tumour specimens. Ninety-three formalin-fixed (43 men, 50 women) paraffin-embedded specimens of primary OSCC cases were randomly selected, which were operated at the Nara Medical University Hospital from 2002 to 2005. Medical records and prognostic follow-up data were obtained from the patient database administered by the hospital. The primary site of the cases was tongue (52 cases), gingiva (27 cases), buccal mucosa ( 9 cases), and other ( 5 case). The tumours were classified according to the International Union Against Cancer TNM classification system (Kreppel et al, 2010).

Immunohistochemistry. Consecutive $3-\mu \mathrm{m}$ sections were cut from each block. Immunohistochemistry was performed by the immunoperoxidase technique, following antigen retrieval by incubation with pepsin (Sigma Chemical, St Louis, MO, USA) for $20 \mathrm{~min}$, specimens were rinsed with phosphate-buffered saline (PBS, Sigma). Antibodies for MIA2 (Abcam, Cambridge, UK), CD3, CD4, CD8, CD20 (DAKO, Carpinteria, CA, USA), CD40L (AbD Serotec, Oxford, UK), granzyme B (Spring Bioscience, Pleasanton, CA, USA), and FOXP3 (Origene Technology, Rockville, $\mathrm{MD}$, USA), diluted at $0.5 \mu \mathrm{g} \mathrm{ml}^{-1}$, were used as primary antibody. After overnight incubation at room temperature, specimens were rinsed with PBS and incubated at room temperature for $1 \mathrm{~h}$ with secondary antibody conjugated to peroxidase $\left(0.2 \mu \mathrm{g} \mathrm{ml}^{-1}\right)$. The specimens were then rinsed with PBS and colour developed in the presence diaminobenzidine (DAB) solution (DAKO). After washing, specimens were counterstained with Meyer's haematoxyline (Sigma). Immunostaining of all samples was performed at the same conditions of antibody reaction and $\mathrm{DAB}$ exposure.

Evaluation of immunohistochemistry. The intensity of immunoreactivity for MIA2 was classified with four grades: grade 0 was no immunoreactivity, grade 1 was weak, grade 2 was moderate, and grade 3 was strong immunoreactivity (Figure 1C-F). Cases with grade 2-3 immunoreactivity were judged as positive for MIA2 expression. Infiltration pattern of lymphocytes was classified in three patterns (Figure 3). Grade $\mathrm{A}$ is the scattered infiltration of lymphocytes at only the outside of tumour nests. Grade B is intermediate infiltration of lymphocytes at the outside and inside of tumour nests. Grade C is marked infiltration of lymphocytes at the outside and inside of tumour nests.

Cell lines and cell culture. HSC3 (drives from metastatic focus of human tongue SCC), HSC4 (derived from primary tumour of the same case of HSC3), and KON (derived from nodal metastatic focus of human oral floor cell lines) were purchase from Health Science Research Resources Bank (Osaka, Japan) were used. MOLT-3 T-lymphoblastic leukaemia cell line was purchased from Dainihon Pharmaceutical, Tokyo, Japan. Cells were maintained in Dulbecco's modified Eagle's medium (DMEM) (Wako Pure Chemical, Osaka, Japan) supplemented with 10\% FBS (Sigma) in $5 \% \mathrm{CO}_{2}$ and $95 \%$ air at $37^{\circ} \mathrm{C}$. For MIA2 inhibition, antibody to MIA2 (Abcam, $1 \mu \mathrm{g} \mathrm{ml}^{-1}$ ) was added to culture medium and cultured for $48 \mathrm{~h}$. For the control, rabbit serum (DAKO, $1 \mu \mathrm{g} \mathrm{ml}^{-1}$ ) was used. Extracellular signal-regulated kinase (ERK) inhibitor (Calbiochem-Novabiochem, Darmstadt, Germany), p38 mitogen-activated protein kinase (MAPK) inhibitor (SB239063, Sigma), and c-Jun N-terminal kinase (JNK) inhibitor (SP600125, Biomol, Hamburg, Germany) were treated for $24 \mathrm{~h}$. Apoptosis were assessed by staining with Hoechst33258 fluorescent dye (Wako). Number of apoptotic cells was counted by observation of 500 cells.

Coculture of OSCC cell with T lymphocytes. HSC3 cells and $\left(5 \times 10^{2}\right)$ and MOLT- 4 cells $\left(5 \times 10^{2}\right)$ were seeded and cultured for $24 \mathrm{~h}$ in 96 -well culture dish. Cells fixed with $4 \%$ paraformaldehyde for $6 \mathrm{~h}$ at $4{ }^{\circ} \mathrm{C}$ were treated with peroxidase-conjugated anti-CD3 antibody (DAKO, $0.5 \mu \mathrm{g} \mathrm{ml}^{-1}$ ) for $30 \mathrm{~min}$ at $37^{\circ} \mathrm{C}$. Cells were treated with tetramethylbenzidin (DAKO) after rinse with PBS for colour development. The reaction was stopped with $0.2 \mathrm{~N} \mathrm{H}_{2} \mathrm{SO}_{4}$ and the colour concentration was measured at OD $450 \mathrm{~nm}$. AntiMIA2 antibody (Abcam) was used for antibody treatment with a concentration of $0.5 \mu \mathrm{g} \mathrm{ml}^{-1}$.

Short interfering RNA. Stealth Select RNAi (short interfering RNA, siRNA) for MIA, MIA2, ITGA4 and ITGA5, and negative siRNA (control siRNA) were purchased from Invitrogen (Carlsbad, CA, USA). Short interfering RNAs (20 nM) were transfected with Lipofectamine2000 (Invitrogen) according to the manufacture's recommendations. Effect of siRNA was confirmed by real-time RT-PCR.

Transwell infiltration assay of $\mathbf{T}$ lymphocytes. Our previous method was modified (Kuniyasu et al, 2004). HSC3 cells $\left(1 \times 10^{4}\right)$ were seeded on the bottom of the insert well coated with fibronectin (pore size $3 \mu \mathrm{m}$; diameter $5 \mathrm{~mm}$, Becton-Dickinson, Bedford, MA, USA), which was set on the well and exchanged medium to DMEM with $2 \%$ bovine serum albumin (BSA; Sigma). MOLT-3 cells $\left(5 \times 10^{3}\right)$, which were surface-labelled with PKH26 chemifluorescent dye (Zynaxis, Malvern, PA, USA), were seeded on each insert using 2\% BSA-DMEM. After 6-h incubation, infiltrating MOLT-3 cells were counted as fluorescence-positive cells in the lower chamber by an autocytometer (Sysmecs, Kobe, Japan).

Quantitative reverse transcription-PCR. The extraction of total RNA was carried out using RNeasy Mini Kit (Qiagen Genomics, Bothell, WA, USA), and total RNA ( $1 \mu \mathrm{g})$ was synthesised with the ReverTra Ace- $\alpha$-RT Kit (Toyobo, Osaka, Japan). Quantitative reverse transcription-PCR (qRT-PCR) was performed by StepOne Real-Time PCR Systems (Applied Biosystems, Foster City, CA, USA) using Fast SYBR Green Master Mix (Applied Biosystems), and analysed by the relative standard curve quantification method (Livak and Schmittgen, 2001). PCR condition was set according to 

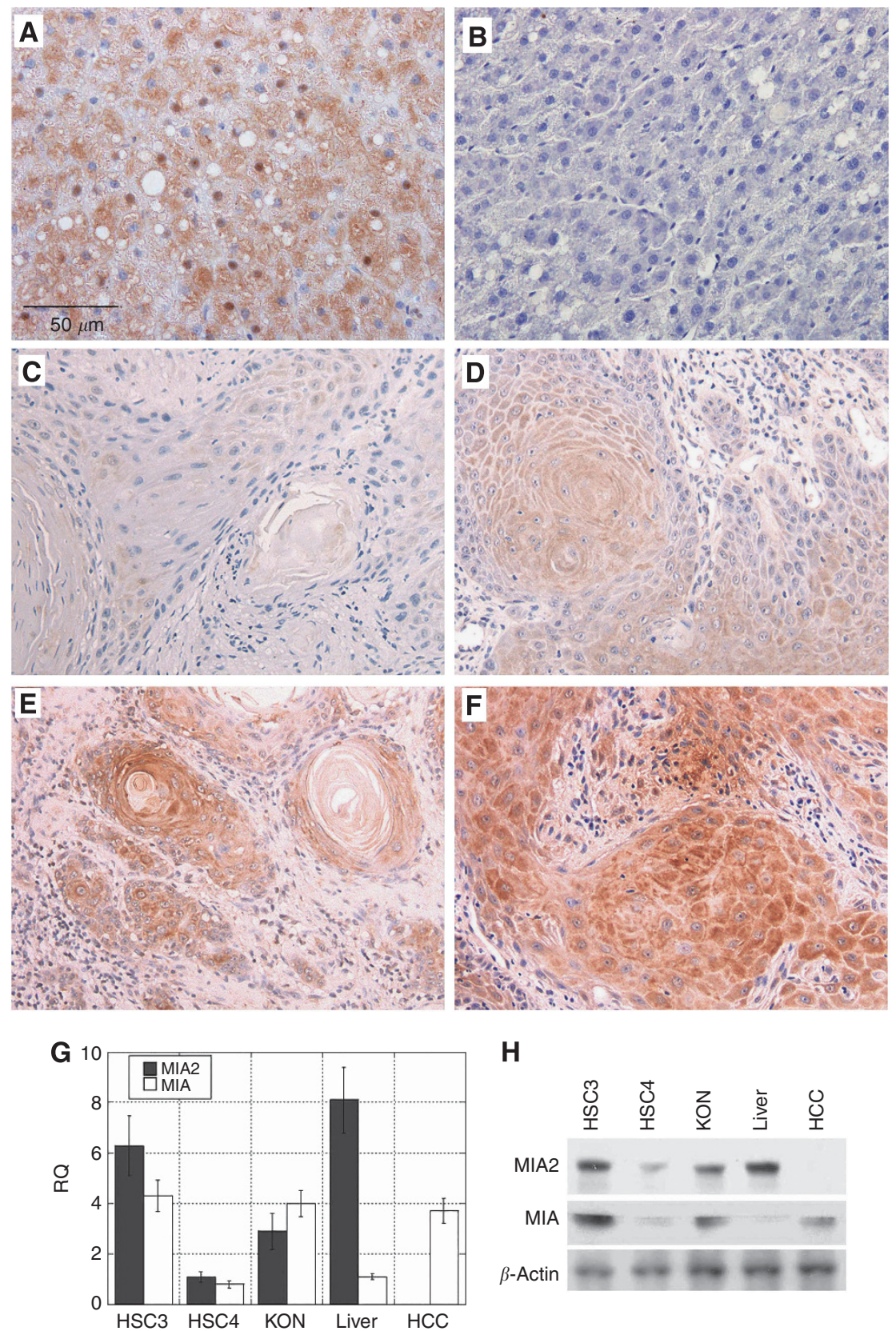

H

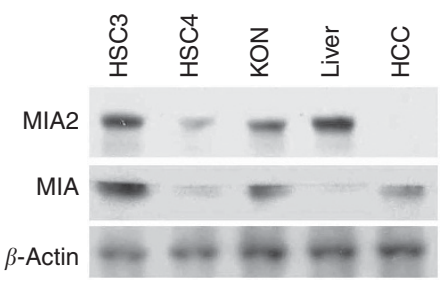

Figure 1. Expression of melanoma inhibitory activity 2 (MIA2) in oral squamous cell carcinoma (OSCC). (A) MIA2 immunoreactivity in a non-tumorous, non-hepatitis liver specimen that was used as a positive control. The cytoplasm of the hepatocytes in an acute hepatitis case showed high-grade MIA2 immunoreactivity. (B) A specimen of human hepatocellular carcinoma was examined as a negative control. It showed no MIA2 immunoreactivity. (C-F) Immunohistochemical analysis of MIA2 expression in OSCC cases. Positive immunoreactivity was seen in the cytoplasm of the cancer cells. (C) A well-differentiated stage I OSCC case (T1 NO MO) showed no MIA2 immunoreactivity (grade 0). (D) A well-differentiated stage II OSCC case (T2 NO MO) showed weak MIA2 immunoreactivity (grade 1). (E) A well-differentiated stage IV OSCC case (T4 NO MO) showed moderate MIA2 immunoreactivity (grade 2). (F) A well-differentiated stage III OSCC case (T3 N1 MO) showed strong MIA2 immunoreactivity (grade 3). Bar, $50 \mu \mathrm{m}$. MIA2 and MIA mRNA expressions were examined using qRT-PCR (G) and immunoblotting $(\mathbf{H})$ in the highly metastatic cell line HSC3, low metastatic cell line HSC4, and KON cell line derived from metastatic lymph nodes cells. The tissues of non-tumorous, non-hepatitis liver and hepatocellular carcinoma were examined as positive and negative control, respectively, for MIA2 expression. Melanoma inhibitory activity expression was also examined. $\beta$-Actin served as the loading control.

the provider's instructions. ACTB mRNA was amplified for internal control (GenBank accession No. NM 001101). Each amplification reaction was evaluated by a melting curve analysis. For visualising PCR products, agarose gel electrophoresis was performed with ethidium bromide staining.

Preparation of conditioned medium. HSC3 cells were cultured in $2 \%$ BSA-DMEM for $12 \mathrm{~h}$ by treatment with MIA2 siRNA or
anti-MIA2 antibody (Abcam, $0.5 \mu \mathrm{g} \mathrm{ml}^{-1}$ ). Then the conditioned medium was filtered with a $0.2-\mu \mathrm{m}$-filter (Becton-Dickinson Labware).

Immunoblotting. Cell lysate was extracted as previously described (Kuniyasu et al, 2002). Cell lysate $(50 \mu \mathrm{g})$ was separated by $12.5 \%$ SDS-PAGE. Proteins were blotted onto nitrocellulose membrane electronically. The membrane was treated with primary 


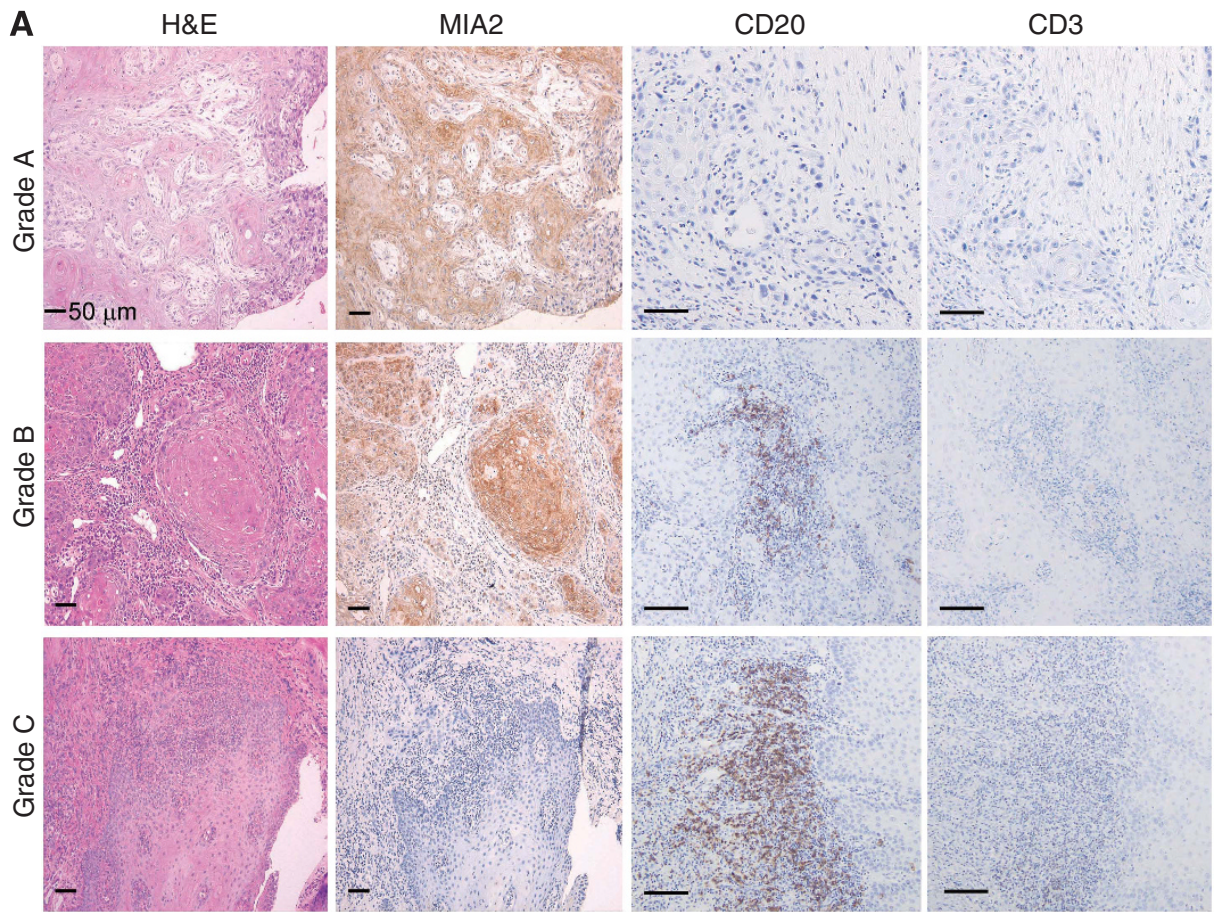

B
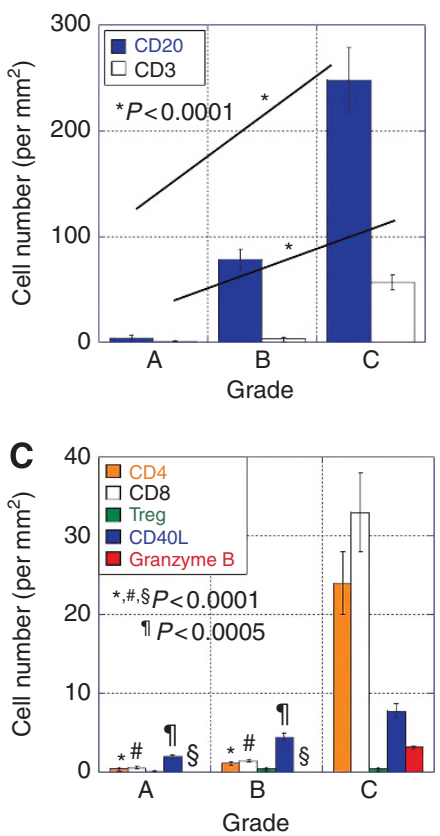

Figure 2. Infiltration pattern of lymphocytes in oral squamous cell carcinoma (OSCC). (A) The infiltration pattern of lymphocytes in OSCC was classified into three patterns: grade A, scattered infiltration of lymphocytes at only the outside of the tumour nests; grade B, intermediate infiltration of lymphocytes at the outside and inside of tumour nests; and grade $\mathrm{C}$, marked infiltration of lymphocytes at the outside and inside of the tumour nests. Bar, $50 \mu \mathrm{m}$. Melanoma inhibitory activity 2 expression and $\mathrm{CD} 2 \mathrm{O}(+)$ and $\mathrm{CD} 3(+)$ lymphocytes were examined by immunohistochemistry in each case. (B) Number of infiltrating CD3(+) and CD2O(+) cells in tumours of 93 OSCC cases. (C) Number of infiltrating $\mathrm{CD} 4(+), \mathrm{CD} 8(+), \mathrm{FOXP3}(+)$ regulatory T cell (Treg), and CD4OL(+)-activated CD4 cells in tumours of 93 OSCC cases. Error bar, s.d.

antibodies to MIA and MIA2 (Abcam), integrin $\alpha 4$, integrin $\alpha 5$, phosphorylated ERK1/2, phosphorylated JNK, phosphorylated p38, VEGF, VEGF-C, and VEGF-D (Santa-Cruz Biotechnology, Santa-Cruz, CA, USA). An $\alpha$-tubulin antibody was used to assess the levels of protein loaded per lane (Oncogene Research Products, Cambridge, MA, USA). The immune complex was visualised with an ECL western-blot detection system (Amersham, Aylesbury, $\mathrm{UK})$.

Immunoprecipitation. Immunoprecipitation was performed according to the method described previously (Kuniyasu et al, 2001). The lysates were pre-cleaned in lysis buffer with protein A/ $\mathrm{G}$ agarose (Santa-Cruz) for $1 \mathrm{~h}$ at $4{ }^{\circ} \mathrm{C}$ and subsequently centrifuged. The supernatants were incubated with precipitation antibody and protein $\mathrm{A} / \mathrm{G}$ agarose for $3 \mathrm{~h}$ at $4{ }^{\circ} \mathrm{C}$. Precipitates were collected by centrifugation and washed five times with lysis buffer. Precipitates solubilised with sample buffer (Sigma, $40 \mu \mathrm{g}$ ) were subjected to immunoblot analysis. For precipitation, antibodies to integrin $\alpha 4$ and integrin $\alpha 5$ (Santa-Cruz) were used. For detection, antibodies to MIA and MIA2 (Abcam) were used.

Statistical analysis. Statistical analysis of experimental data was done by Mann-Whitney $U$-test and $\chi^{2}$ test. The statistical significance was defined as a two-sided $P$-value of $<0.05$.

\section{RESULTS}

MIA2 expression in OSCC. MIA2 expression was examined by immunohistochemistry in 93 cases of OSCC (Table 1). Melanoma inhibitory activity 2 immunoreactivity was detected in the cytoplasm of hepatocytes and cancer cells of OSCC (Figure 1A and $\mathrm{C}-\mathrm{F}$ ). In contrast, HCC cells did not show MIA2 immunoreactivity (Figure 1B). Cases with grade 2 or 3 immunoreactivity were judged as positive for MIA2 expression.
We next compared the MIA2 expression in HSC3 (high metastatic cell line), HSC4 (low metastatic cell line), and KON (metastatic lymph nodes cell line) using qRT-PCR and immunobloting (Figure $1 \mathrm{G}$ and $\mathrm{H}$ ). The MIA2 expression level was higher in HSC 3 than in HSC4 and KON. The MIA2 expression in OSCC cells was compared with the MIA expression. The MIA2 and MIA were expressed in all OSCC cell lines at various levels.

Relation of MIA2 expression with clinicopathological parameters. MIA2 expression was observed in $62(66.7 \%)$ of the 93 cases and was associated with $\mathrm{T}$ classification (tumour expansion) and nodal metastasis (Table 1). Of the 22 cases of local invasion (T3 or T4), 14 (63.6\%) expressed MIA2, whereas only $18(25.3 \%)$ of the 71 cases of early cancer (T1 or T2) expressed MIA2 $(P=0.0017)$. Of the 30 cases with nodal metastasis $(n+), 17$ (56.7\%) showed MIA2 expression, whereas only 15 (23.8\%) of the 63 cases without nodal metastasis $(n-)$ expressed MIA2 $(P=0.0025)$. However, no significant relationship was found between MIA2 grading and the other parameters including age, sex, primary site, clinical stage, histological differentiation, and tumour recurrence.

MIA2 expression and lymphocyte infiltration. The infiltration pattern of the lymphocytes was classified into three patterns (Figure 2A). Grade $\mathrm{C}$ showed the most pronounced lymphocyte infiltration among the three patterns, whereas grade A showed mild and remote lymphocytes infiltration. As shown in Table 2, MIA2 expression was inversely associated with lymphocyte infiltration. Of the 31 grade A cases, 17 (54.8\%) showed MIA2 expression, whereas only $4(12.9 \%)$ of the 31 grade $\mathrm{C}$ cases expressed MIA2. This result suggests that MIA2 might inhibit intratumoral lymphocyte infiltration.

To examine the detail of MIA2 effect on lymphocytes, subpopulation of them was examined (Figure $2 \mathrm{~A}-\mathrm{C}$ ). $\mathrm{CD} 3+$ or $\mathrm{CD} 20+$ lymphocytes were markedly decreased in grade A case 


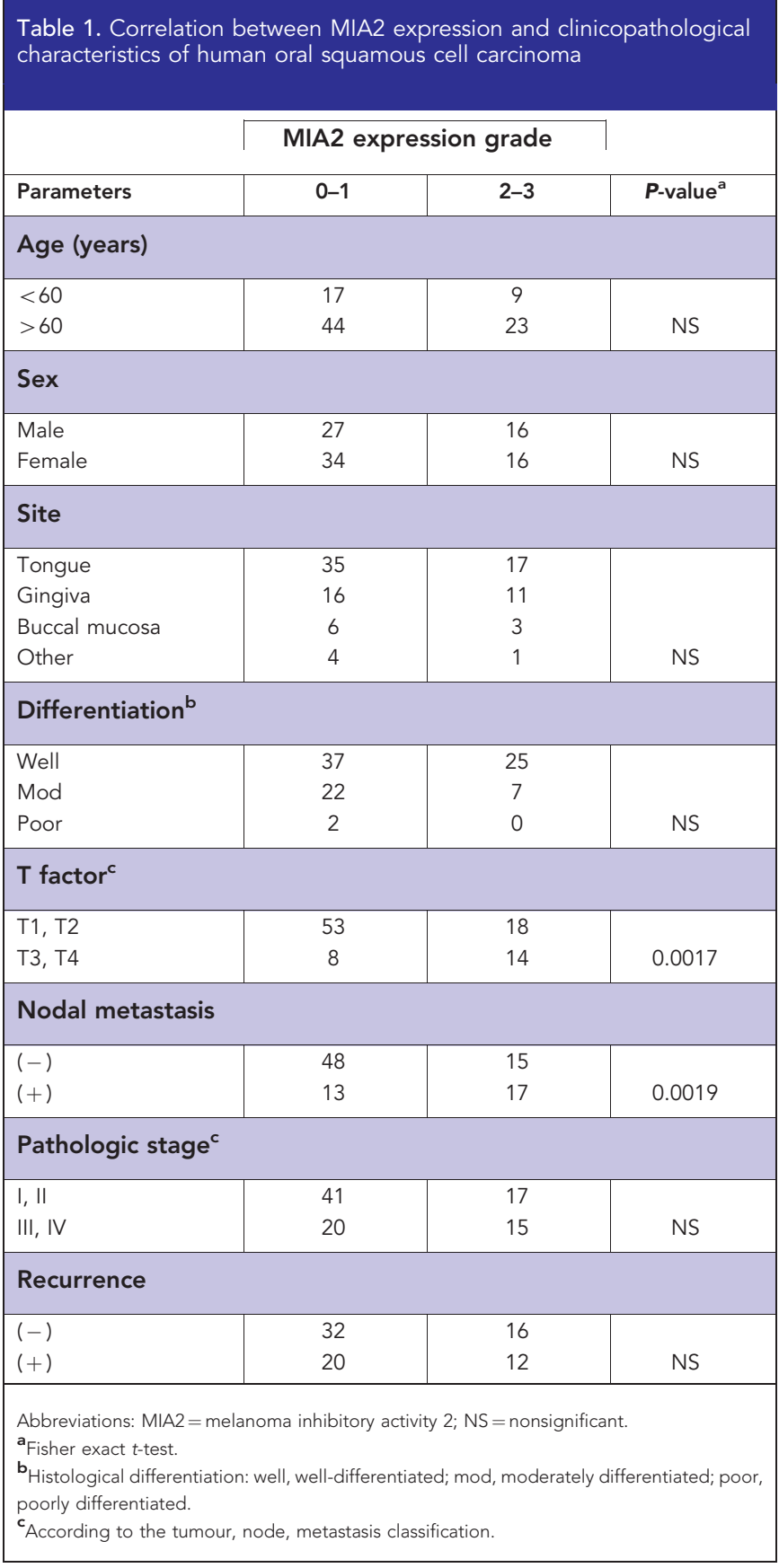

Table 2. Relationship between MIA2 expression and infiltration pattern of lymphocytes in oral squamous cell carcinoma

\begin{tabular}{|l|c|c|}
\cline { 2 - 3 } \multicolumn{2}{c|}{} & \multicolumn{2}{c|}{ MIA2 expression } \\
\hline Lymphocyte infiltration pattern & Grade 0-1 & Grade 2-3 \\
\hline Grade A & $14(23 \%)$ & $17(53 \%)$ \\
\hline Grade B & $20(33 \%)$ & $11(34 \%)$ \\
\hline Grade C & $27(44 \%)$ & $4(13 \%)$ \\
\hline Abbreviation: MIA2 = melanoma inhibitory activity 2. $P=0.00248\left(x^{2}\right.$ test). \\
\hline
\end{tabular}

(Figure 2A). As shown in Figure 2B, both CD3 + and CD20+ lymphocytes were decreased as the grade; however, decrease of $\mathrm{CD} 3+$ lymphocytes was more pronounced than that of CD20 lymphocytes. Next the sub-population of $\mathrm{T}$ lymphocytes was examined (Figure 2C). In grade $\mathrm{A}$ and $\mathrm{B}, \mathrm{CD} 4+, \mathrm{CD} 8+$, and granzyme $\mathrm{B}+\mathrm{T}$ lymphocytes were markedly decreased than that in grade C; however, CD40L + lymphocytes showed more modest association with the grade. The number of regulatory T lymphocytes was not associated with the grade.

Effect of MIA2 knockdown in the OSCC cell lines. We next performed MIA2 knockdown by siRNA treatment to examine the roles of MIA2 on cell growth, invasion, and apoptosis in HSC3 cells (Figure 3 ). Melanoma inhibitory activity 2 siRNA treatment decreased the levels of mRNA and protein in HSC3 cells (Figure 3A). Compared with the control siRNA treatment, cell invasion and anti-apoptotic survival were reduced by MIA2 siRNA treatment $(P=0.0417$ and $P=0.018$, respectively). Cell growth with MIA2 siRNA treatment was not significantly different from that before the treatment.

Effect of MIA2 depletion on lymphocytes infiltration. HSC3 cells and MOLT-3 lymphocytes were cocultured to confirm the lymphocyte inhibitory effect of MIA2 (Figure 4A). The number of MOLT-3 cells was not affected by the coculturing. Next, we conducted a trans-cell layer assay using an insert chamber (Figure 4C-D). The MIA2 protein levels in the cultured medium of HSC3 cell were examined (Figure 4B). Melanoma inhibitory activity 2 knockdown and absorption by anti-MIA2 antibody depleted MIA2 protein in the medium. The number of MOLT-3 lymphocytes that passed through the HSC3 cell layer at the bottom of the insert was then counted. The infiltrating cell number was increased by MIA2 depletion by MIA2 knockdown and the antibody treatment.

Effect of MIA2 knockdown on the VEGF, VEGF-C, and VEGF-D expressions in the HSC3 OSCC cells. MIA is a known angiogenic and lymphangiogenic factor inducing VEGF and VEGF-D (Sasahira et al, 2008, 2010). As such, we examined the effect of MIA2 knockdown on the levels of mRNA and protein of VEGF, VEGF-C, and VEGF-D and compared it with the effect of MIA knockdown in HSC3 cells. Treatment with specific siRNAs to MIA2 or MIA decreased the levels of mRNA and protein in the HSC3 cells (Figure 5A and B). The HSC3 cells after MIA2 knockdown showed modestly inhibited VEGF and VEGF-C expressions compared with those after MIA knockdown (Figure 5C and D). In contrast, the HSC3 cells after MIA2 knockdown showed VEGF-D downregulation similar to that in cells after MIA knockdown (Figure 5E). It is interesting that the double knockdown of MIA2 and MIA showed less of an inhibitory effect on VEGF and VEGF-C downregulation, whereas it showed a greater inhibitory effect on VEGF-D downregulation (Figure 5C-E). Finally, the HSC3 cells after MIA or MIA2 knockdown were treated with ERK1/2 and p38 inhibitors (Figure 5F). In both MIA and MIA2 knockdown, VEGF-C expression was decreased by p38 inhibition, whereas VEGF-D was decreased by ERK1/2 inhibition.

Comparison of the role of integrins in MIA2 function. MIA is a known interacting partner of integrins $\alpha 4$ and $\alpha 5$ (Bauer et al, 2006). As such, we examined the physical association between MIA2 and the integrins (Figure 6A). The HSC3 lysates showed coprecipitation between MIA2 and integrin $\alpha 4$ or $\alpha 5$ as with MIA. Melanoma inhibitory activity 2 showed higher affinity with integrin $\alpha 4$ than that with integrin $\alpha 5$, unlike MIA in the HSC3 cells. Knockdown of the expression of integrins $\alpha 4$ (ITGA4) and $\alpha 5$ (ITGA5) was confirmed in the HSC3 cells (Figure 1B). Using the knockdown system, the phosphorylation status of the MAPK family was examined (Figure 6C-E). In the MIA-knockdown HSC3 cells, which expressed MIA2, ITGA4 knockdown decreased p38 phosphorylation. In contrast, ITGA5 knockdown decreased the phosphorylation of p38 and JNK. In MIA2-knockdown HSC3 cells 

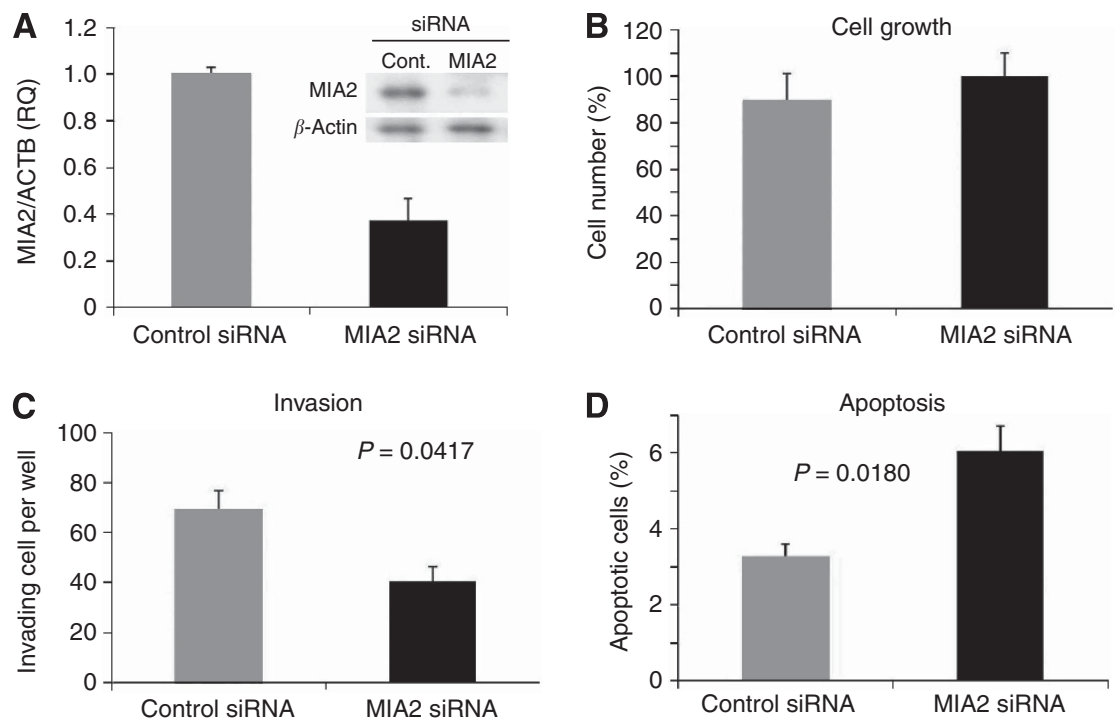

Figure 3. Effect of knockdown of melanoma inhibitory activity 2 (MIA2) in oral squamous cell carcinoma (OSCC) cell lines. (A) The effect of MIA2 siRNA treatment on MIA2 mRNA expression in HSC3 cells. Melanoma inhibitory activity 2 protein levels were also shown in the inset. The effects of MIA2 knockdown on cell growth (B), invasion (C), and apoptosis (D) in the HSC3 cells. Error bar, s.d.

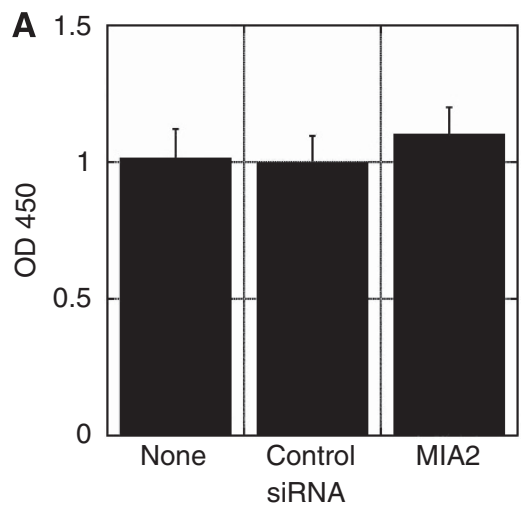

B
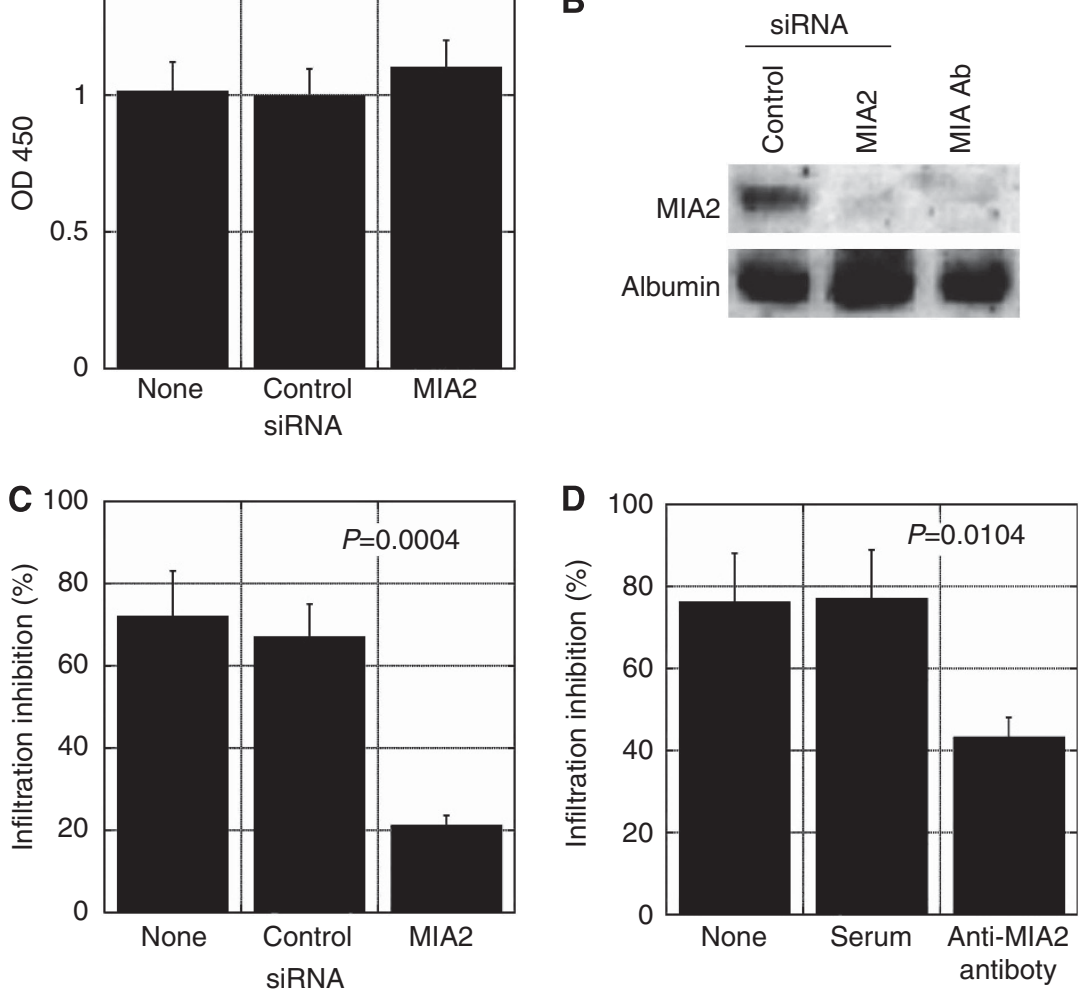

Figure 4. Transwell infiltration of MOLT-3 lymphocytes through an HSC3 cell layer. (A) The HSC3 and MOLT-3 cells were cocultured with melanoma inhibitory activity 2 (MIA2) siRNA or control siRNA. (C, D) Inhibition of transwell infiltration of MOLT-3 cells. The number of MOLT-3 cells infiltrating through the HSC3 cell layer at the bottom of the insert was compared with that using the insert without cell layer. (C) The cells were exposed to MIA2 siRNA or control siRNA. (B) The cells were treated with control serum or anti-MIA2 antibody. Error bar, s.d.

expressing MIA, knockdown of ITGA4 and ITGA5 decreased the phosphorylation of p38 and JNK.

Finally, we examined the effect of ITGA4 or ITGA5 knockdown and MAPK inhibitors on apoptosis in MIA-knockdown HSC3 cells (Figure 6F and G). ITGA4 knockdown increased apoptosis, whereas ITGA5 knockdown decreased apoptosis. The inhibition of JNK decreased apoptosis, whereas inhibition of p38 increased apoptosis. These findings suggest that JNK activation by MIA2 through integrin $\alpha 5$ might induce apoptosis. In contrast, p38 activation through integrin $\alpha 4$ and $\alpha 5$ might suppress apoptosis. 

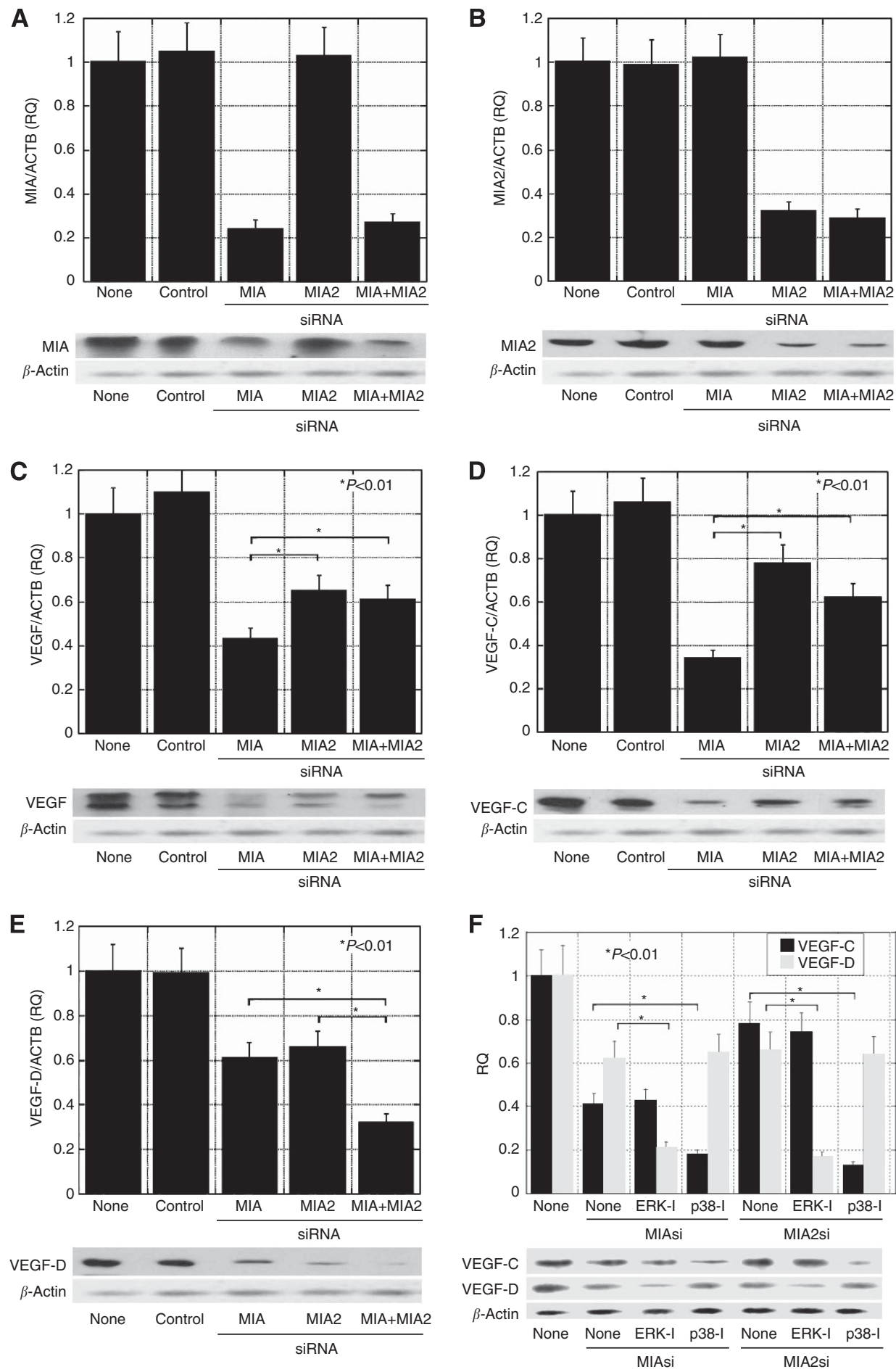

Figure 5. Effect of knockdown of melanoma inhibitory activity 2 (MIA2) and/or MIA on vascular endothelial growth factor (VEGF), VEGF-C, and VEGF-D expressions in the HSC3 cells. Effect of knockdown of MIA and/or MIA2 on the expression of MIA (A) and MIA2 (B) was confirmed using qRT-PCR and immunoblotting. The expressions of VEGF (C), VEGF-C (D), and VEGF-D (E) were examined by qRT-PCR and immunoblotting in HSC3 cells with knockdown of MIA, MIA2, or MIA + MIA2. (F) Expression of VEGF-C and -D was examined using qRT-PCR and immunoblotting in MIA- or MIA2-knockdown HSC3 cells treated with inhibitors of ERK1/2 (ERK-I) or p38 (p38-I). Error bar, s.d.

\section{DISCUSSION}

MIA2 is isolated as a second member of the MIA homologous gene family. Melanoma inhibitory activity is a protumoral factor in the progression and metastasis of malignant melanoma and OSCC. In contrast, MIA2 is downregulated in HCC, which suggests that MIA2 has a putative tumour-suppressive function. It is interesting that our data on OSCC suggest that MIA2 might act as a protumoral factor similar to MIA.

In clinical study, MIA2 expression was associated with tumour expansion, nodal metastasis, less lymphocyte infiltration, and advanced-stage OSCC. Highly metastatic OSCC cell lines expressed MIA2 at higher levels than a low metastatic cell line. Melanoma inhibitory activity 2 knockdown was associated with inhibition of cell invasion, anti-apoptotic survival, and VEGF, VEGF-C, and 
A

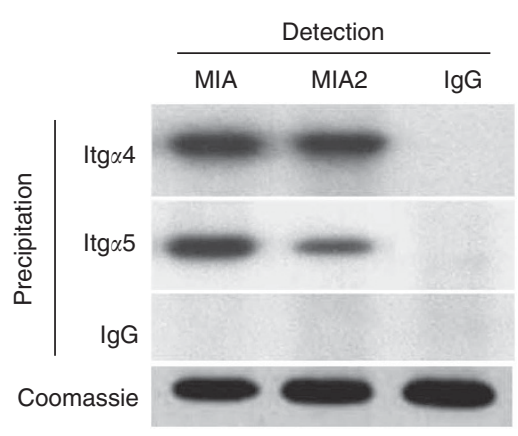

C
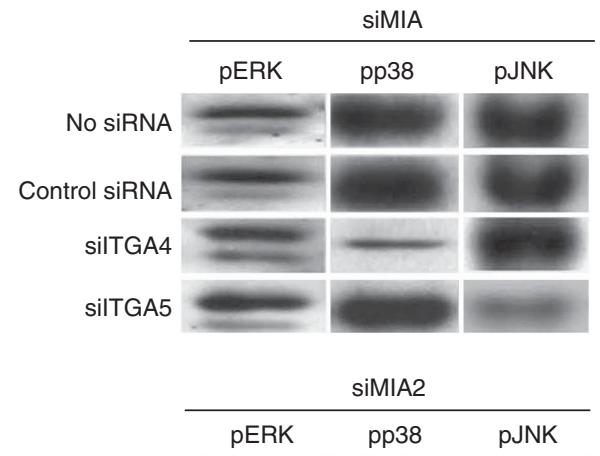

No siRNA

Control siRNA

silTGA4

silTGA5
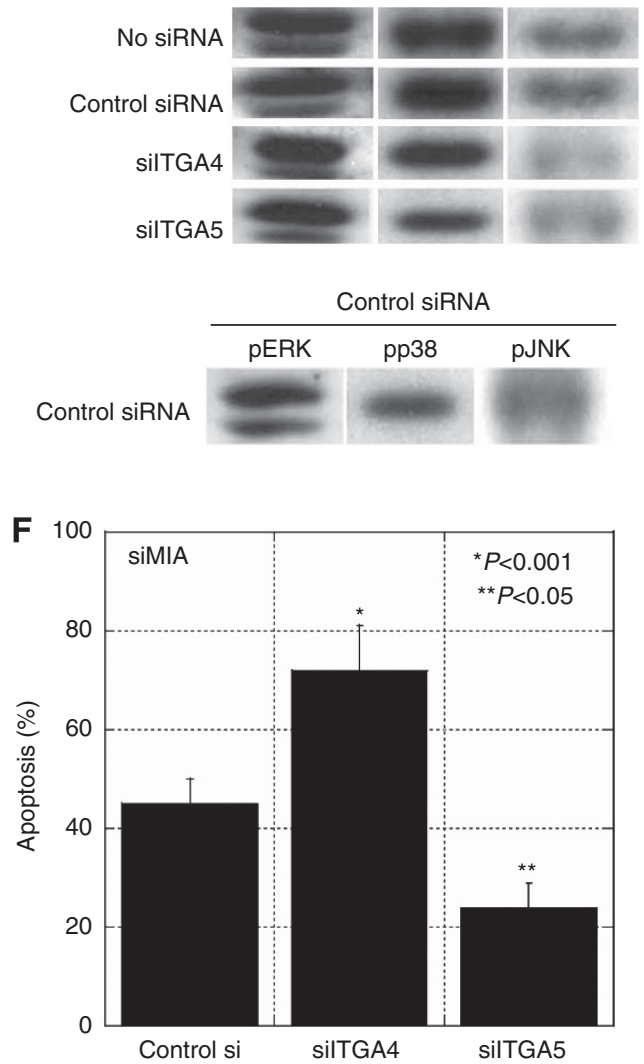
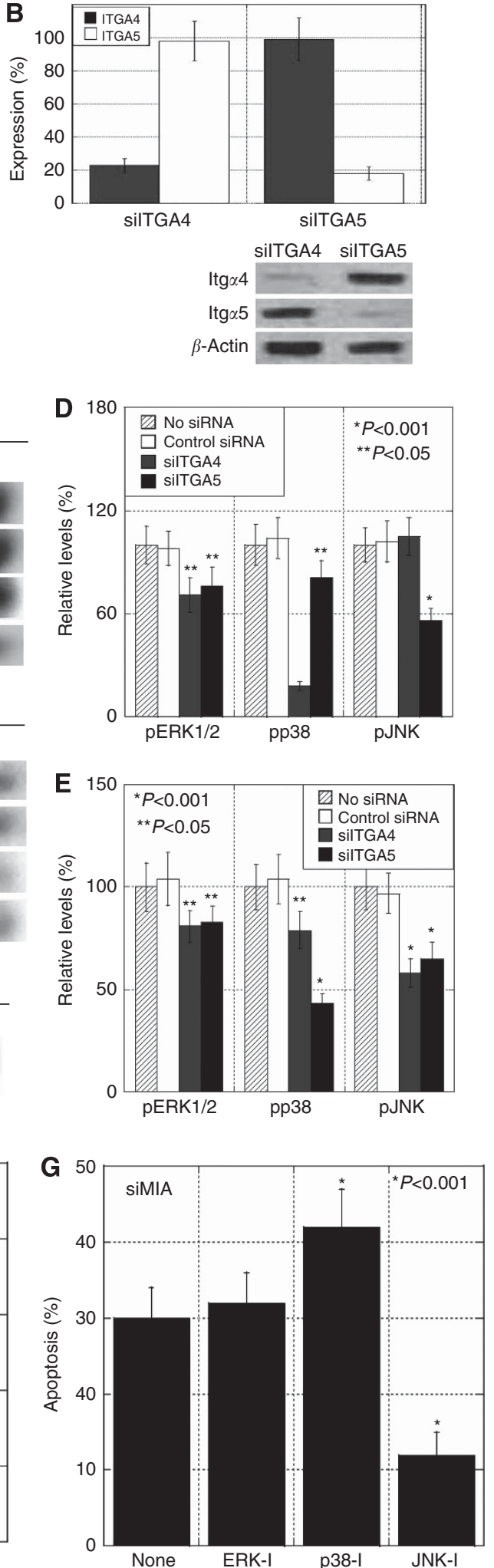

Figure 6. Roles of integrin and mitogen-activated protein kinase (MAPK) in melanoma inhibitory activity 2 (MIA2) function. Immunoprecipitation of the HSC3 cell lysate precipitated with antibodies to integrin $\alpha 4$ (Itg $\alpha 4)$ or integrin $\alpha 5(\lg \alpha 5)$, detected with antibodies to MIA or MIA2. Precipitation and detection by lgG was done for negative control. Coomassie blue staining of slot blot samples served as a control for protein content. (B) Knockdown of integrin $\alpha 4$ (ITGA4) or integrin $\alpha 5$ (ITGA5) was examined using qRT-PCR and immunoblotting. (C) Phosphorylation levels of MAPK families were examined by immunoblotting in HSC3 cells with concurrent knockdown of MIA or MIA2 and ITGA4 or ITGA5. (D, E) Phosphorylation levels of the MAPK families were semiquantified with standardisation to those in HSC3 cells untreated with siRNA. $(\mathbf{F}, \mathbf{G})$ Effect of knockdown of ITGA4 or ITGA5, p38 inhibitor (p38-I), or JNK (JNK-I) on trichostatin A-induced apoptosis. The percentage of apoptotic cells to 500 counted cells was calculated. Error bar, s.d. 
VEGF-D expressions. These results suggest that MIA2 increases invasion, survival and angiogenesis, and inhibits host anticancer immunity.

MIA2 shares protein homology, including src homology domain-3, with MIA (Bosserhoff et al, 2004). The immunoprecipitation assay in the present study showed that MIA2 and MIA are bound to integrins $\alpha 4$ and $\alpha 5$; however, MIA2 showed higher affinity to integrin $\alpha 4$ than to integrin $\alpha 5$. Melanoma inhibitory activity activated p38 and JNK through integrins $\alpha 4$ and $\alpha 5$, whereas MIA2 activated p38 through integrin $\alpha 4$ and $\alpha 5$, and activated JNK through integrin $\alpha 5$. Moreover, the MIA2-integrin $\alpha 5$-JNK signal increased apoptosis, whereas the MIA2-integrin a4-p38 signal decreased apoptosis.

MIA2 had a pro-angiogenic role by increasing VEGF family expression. The increment was modest compared with that by MIA; however, MIA2 showed a less pronounced increase in VEGD and VEGF-C than that by MIA. In contrast, VEGF-D upregulation by MIA2 was at the same level to that by MIA. The VEGF-C upregulation signal by MIA2 was carried through p38, which is also associated with MIA-induced VEGF family upregulation (Sasahira et al, 2008, 2010). In contrast, the VEGF-D upregulation signal by MIA2 was carried through ERK1/2 as found in that by MIA (Sasahira et al, 2008). Coexpressed MIA and MIA2 was found in most OSCC cell lines and human OSCC tumours, and expression of the VEGF family might be affected by MIA and MIA2 by a synergic (as show in VEGF-D) or nonsynergic (as shown in VEGF and VEGF-C) manner.

MIA2 expression was inversely associated with lymphocyte infiltration into the tumours. Melanoma inhibitory activity binds to integrin $\alpha 4$, which is expressed in the host immune cells and suppresses lymphokine-activated killer cell cytotoxicity (Jachimczak et al, 2005). Our data showed that MIA2 also bound to integrin $\alpha 4$, which might inhibit lymphocyte infiltration into the tumour. The coculture of HSC3 cells and MOLT-3 lymphocytes did not induce cell death in MOLT-3 cells, an observation suggesting that MIA2 possesses no cytotoxic effect on lymphocytes. As MIA binds to fibronectin to induce tumour cell detachment from the extracellular matrix (Bosserhoff et al, 2003b), MIA2 might also bind to fibronectin. Fibronectin induces chemotaxis of $\mathrm{T}$ lymphocytes in combination with stromal cell-derived factor $1 \alpha$ (Savino et al, 2002). The masking of fibronectin by MIA or MIA2 might inhibit chemotaxis of the T lymphocytes.

Among lymphocyte sub-population, decrease of $\mathrm{CD} 4+$, $\mathrm{CD} 8+$, and granzyme $\mathrm{B}+$ lymphocytes was most significantly associated with the grade, and subsequently with MIA2 expression, which suggest that MIA2 might inhibit cytotoxic T lymphocytes mainly. No alteration in the number of regulatory $\mathrm{T}$ lymphocytes might enhance their immune-suppressive effect through decrease of other types of $\mathrm{T}$ lymphocytes. Importantly, CD20 + B lymphocytes also showed a decrease in association with grade or MIA2 expression. The decrease in association of CD40L + $\mathrm{T}$ lymphocytes, which are associated with humoral immune activity, might decrease the association of $\mathrm{B}$ lymphocytes (Lederman et al, 1996).

MIA is regulated by HMGB1 via a highly conserved region promoter element in the $5^{\prime}$-flanking region of the MIA gene (Poser et al, 2003). We reported previously that HMGB1 affected MIA expression in OSCC cells (Sasahira et al, 2008). Comparison of MIA and MIA2 protein levels showed no association between them. Therefore, MIA2 might not be regulated by HMGB1. In the liver, MIA2 expression is transcriptionally regulated by HNF-1 (Bosserhoff et al, 2003a, 2004; Hellerbrand et al, 2008). Hepatocyte nuclear factor-1A expression is reported in human OSCC (O’Donnell et al, 2005), suggesting that it induces MIA2 expression in OSCC. Prostaglandin E2 (PGE2) activates T-cell factor (same as HNF)-dependent transcription (Shao et al, 2005). Prostaglandin E2 is highly involved in tumour growth in the head and neck cancers
(Abrahao et al, 2010). Therefore, MIA2 might be a common tumour-associated factor in OSCC.

Melanoma inhibitory activity 2 is expressed at high levels and acts as an antitumor factor in the liver (Bosserhoff et al, 2004; Hellerbrand et al, 2005, 2008). Melanoma inhibitory activity 2 expression is repressed in hepatitis, cirrhosis, and hepatoma, which is associated with cell proliferation (Hellerbrand et al, 2008; Xu et al, 2011). In contrast, MIA2 acts as a protumoral factor in OSCC. Melanoma inhibitory activity 2 affinity to the MIA receptors, integrins $\alpha 4$ and $\alpha 5$, suggests that MIA2 might act as a competitive inhibitor of MIA. Otherwise, HCC cells express integrin $\alpha 4$ at a markedly lower level than integrin $\alpha 5$ (Fu et al, 2010). These features in the liver suggest that MIA2 might act as a pro-apoptotic factor through integrin $\alpha 5$. Moreover, OSCC concurrently expressed MIA2 with MIA at high frequency (87\%, data not shown). The overlapping of signals from MIA2 and MIA through integrin $\alpha 4$ and $\alpha 5$ might result a protumoral role in OSCC. Melanoma inhibitory activity is expressed in the liver at low levels (Su et al, 2002). A lack of synergism between MIA and MIA2 also might emphasise a pro-apoptotic role in the liver.

These data suggest that MIA2 might act as a protumoral factor that was influenced by concurrent expression of MIA, receptor integrin expression patterns, and activation pattern of MAPK family in OSCC. The frequent expression in OSCC suggests that MIA2 is a relevant target for cancer treatment.

\section{ACKNOWLEDGEMENTS}

This work was supported in part by Grant-in-Aid for Scientific Research from Japan Society for the Promotion of Science, Japan, and Grant-in-Aid for Scientific Research from Ministry of Health, Labour and Welfare, Japan.

\section{CONFLICT OF INTEREST}

The authors declare no conflict of interest.

\section{REFERENCES}

Abrahao AC, Castilho RM, Squarize CH, Molinolo AA, dos Santos-Pinto Jr D, Gutkind JS (2010) A role for COX2-derived PGE2 and PGE2-receptor subtypes in head and neck squamous carcinoma cell proliferation. Oral Oncol 46(12): 880-887.

Adams RH, Alitalo K (2007) Molecular regulation of angiogenesis and lymphangiogenesis. Nat Rev Mol Cell Biol 8(6): 464-478.

Avraamides CJ, Garmy-Susini B, Varner JA (2008) Integrins in angiogenesis and lymphangiogenesis. Nat Rev Cancer 8(8): 604-617.

Bauer R, Humphries M, Fassler R, Winklmeier A, Craig SE, Bosserhoff AK (2006) Regulation of integrin activity by MIA. J Biol Chem 281(17): 11669-11677.

Beck Jr L, D’Amore PA (1997) Vascular development: cellular and molecular regulation. FASEB J 11(5): 365-373.

Bosserhoff AK, Buettner R (2002) Expression, function and clinical relevance of MIA (melanoma inhibitory activity). Histol Histopathol 17(1): 289-300.

Bosserhoff AK, Moser M, Buettner R (2004) Characterization and expression pattern of the novel MIA homolog TANGO. Gene Expr Patterns 4(4): 473-479.

Bosserhoff AK, Moser M, Scholmerich J, Buettner R, Hellerbrand C (2003a) Specific expression and regulation of the new melanoma inhibitory activity-related gene MIA2 in hepatocytes. J Biol Chem 278(17): 15225-15231.

Bosserhoff AK, Stoll R, Sleeman JP, Bataille F, Buettner R, Holak TA (2003b) Active detachment involves inhibition of cell-matrix contacts of malignant melanoma cells by secretion of melanoma inhibitory activity. Lab Invest 83(11): 1583-1594. 
Ferlay J, Shin HR, Bray F, Forman D, Mathers C, Parkin DM (2010) Estimates of worldwide burden of cancer in 2008: GLOBOCAN 2008. Int J Cancer 127(12): 2893-2917.

Fu BH, Wu ZZ, Qin J (2010) Effects of integrins on laminin chemotaxis by hepatocellular carcinoma cells. Mol Biol Rep 37(3): 1665-1670.

Hellerbrand C, Amann T, Schlegel J, Wild P, Bataille F, Spruss T, Hartmann A, Bosserhoff AK (2008) The novel gene MIA2 acts as a tumour suppressor in hepatocellular carcinoma. Gut 57(2): 243-251.

Hellerbrand C, Bataille F, Schlegel J, Hartmann A, Muhlbauer M, Scholmerich J, Buttner R, Hofstadter F, Bosserhoff AK (2005) In situ expression patterns of melanoma inhibitory activity 2 in healthy and diseased livers. Liver Int 25(2): 357-366.

Jachimczak P, Apfel R, Bosserhoff AK, Fabel K, Hau P, Tschertner I, Wise P, Schlingensiepen KH, Schuler-Thurner B, Bogdahn U (2005) Inhibition of immunosuppressive effects of melanoma-inhibiting activity (MIA) by antisense techniques. Int J Cancer 113(1): 88-92.

Kademani D, Bell RB, Bagheri S, Holmgren E, Dierks E, Potter B, Homer L (2005) Prognostic factors in intraoral squamous cell carcinoma: the influence of histologic grade. J Oral Maxillofac Surg 63(11): 1599-1605.

Kreppel M, Eich HT, Kubler A, Zoller JE, Scheer M (2010) Prognostic value of the sixth edition of the UICC's TNM classification and stage grouping for oral cancer. J Surg Oncol 102(5): 443-449.

Kuniyasu H, Oue N, Wakikawa A, Shigeishi H, Matsutani N, Kuraoka K, Ito R, Yokozaki H, Yasui W (2002) Expression of receptors for advanced glycation end-products (RAGE) is closely associated with the invasive and metastatic activity of gastric cancer. J Pathol 196(2): 163-170.

Kuniyasu H, Sasaki T, Sasahira T, Ohmori H, Takahashi T (2004) Depletion of tumor-infiltrating macrophages is associated with amphoterin expression in colon cancer. Pathobiology 71(3): 129-136.

Kuniyasu H, Yasui W, Pettaway CA, Yano S, Oue N, Tahara E, Fidler IJ (2001) Interferon-alpha prevents selection of doxorubicin-resistant undifferentiated-androgen-insensitive metastatic human prostate cancer cells. Prostate 49(1): 19-29.

Lederman S, Cleary AM, Yellin MJ, Frank DM, Karpusas M, Thomas DW, Chess L (1996) The central role of the CD40-ligand and CD40 pathway in T-lymphocyte-mediated differentiation of B lymphocytes. Curr Opin Hematol 3(1): 77-86.

Livak KJ, Schmittgen TD (2001) Analysis of relative gene expression data using real-time quantitative PCR and the 2( - Delta Delta C(T)) Method. Methods 25(4): 402-408.

Lopez-Graniel CM, Tamez de Leon D, Meneses-Garcia A, Gomez-Ruiz C, Frias-Mendivil M, Granados-Garcia M, Barrera-Franco JL (2001) Tumor angiogenesis as a prognostic factor in oral cavity carcinomas. J Exp Clin Cancer Res 20(4): 463-468.

Matsuda T, Marugame T, Kamo K, Katanoda K, Ajiki W, Sobue T (2012) Cancer incidence and incidence rates in Japan in 2006: based on data from 15 population-based cancer registries in the monitoring of cancer incidence in Japan (MCIJ) project. Jpn J Clin Oncol 42(2): 139-147.

Miyahara M, Tanuma J, Sugihara K, Semba I (2007) Tumor lymphangiogenesis correlates with lymph node metastasis and clinicopathologic parameters in oral squamous cell carcinoma. Cancer 110(6): 1287-1294.
Nagler RM (2002) Molecular aspects of oral cancer. Anticancer Res 22(5): 2977-2980.

O’Donnell RK, Kupferman M, Wei SJ, Singhal S (2005) Squamous cell carcinoma of the oral cavity with lymph node metastasis: NCBI Gene Expression Omnibus.

Poser I, Golob M, Buettner R, Bosserhoff AK (2003) Upregulation of HMG1 leads to melanoma inhibitory activity expression in malignant melanoma cells and contributes to their malignancy phenotype. Mol Cell Biol 23(8): 2991-2998.

Sasahira T, Kirita T, Kurihara M, Yamamoto K, Bhawal UK, Bosserhoff AK, Kuniyasu H (2010) MIA-dependent angiogenesis and lymphangiogenesis are closely associated with progression, nodal metastasis and poor prognosis in tongue squamous cell carcinoma. Eur J Cancer 46(12): 2285-2294.

Sasahira T, Kirita T, Oue N, Bhawal UK, Yamamoto K, Fujii K, Ohmori H, Luo Y, Yasui W, Bosserhoff AK, Kuniyasu H (2008) High mobility group box-1-inducible melanoma inhibitory activity is associated with nodal metastasis and lymphangiogenesis in oral squamous cell carcinoma. Cancer Sci 99(9): 1806-1812.

Savino W, Mendes-da-Cruz DA, Silva JS, Dardenne M, Cotta-de-Almeida V (2002) Intrathymic T-cell migration: a combinatorial interplay of extracellular matrix and chemokines? Trends Immunol 23(6): 305-313.

Shang ZJ, Li JR, Li ZB (2002) Circulating levels of vascular endothelial growth factor in patients with oral squamous cell carcinoma. Int J Oral Maxillofac Surg 31(5): 495-498.

Shao J, Jung C, Liu C, Sheng H (2005) Prostaglandin E2 Stimulates the betacatenin/T cell factor-dependent transcription in colon cancer. J Biol Chem 280(28): 26565-26572.

Shintani S, Li C, Ishikawa T, Mihara M, Nakashiro K, Hamakawa H (2004) Expression of vascular endothelial growth factor A, B, C, and D in oral squamous cell carcinoma. Oral Oncol 40(1): 13-20.

Su AI, Cooke MP, Ching KA, Hakak Y (2002) Large-scale analysis of the human transcriptome (HG-U95A): NCBI Gene Expression Omnibus.

Tsurusaki T, Kanda S, Sakai H, Kanetake H, Saito Y, Alitalo K, Koji T (1999) Vascular endothelial growth factor-C expression in human prostatic carcinoma and its relationship to lymph node metastasis. Br J Cancer 80(1-2): 309-313.

Xu Y, Yang Y, Cai Y, Liu F, Liu Y, Zhu Y, Wu J (2011) The X protein of hepatitis $B$ virus activates hepatoma cell proliferation through repressing melanoma inhibitory activity 2 gene. Biochem Biophys Res Commun 416(3-4): 379-384.

Yonemura Y, Endo Y, Fujita H, Fushida S, Ninomiya I, Bandou E, Taniguchi K, Miwa K, Ohoyama S, Sugiyama K, Sasaki T (1999) Role of vascular endothelial growth factor $\mathrm{C}$ expression in the development of lymph node metastasis in gastric cancer. Clin Cancer Res 5(7): 1823-1829.

This work is published under the standard license to publish agreement. After 12 months the work will become freely available and the license terms will switch to a Creative Commons AttributionNonCommercial-Share Alike 3.0 Unported License. 